\title{
ÍNDICE DE INFECÇÃO DE TRIATOMÍNEOS PELO Trypanosoma cruzi, CAPTURADOS NO AMBIENTE DOMICILIAR NO ESTADO DE GOIÁS EM 1993.
}

\author{
Carmeci Natalina Elias**, Ionizete Garcia da Silva , Marlene de Fátima \\ Camargo**, Rosa de Belem das Neves Alves*.
}

\section{RESUMO}

No Estado de Goiás, os trabalhos operacionais sobre o vetor da doença de Chagas, estão distribuídos em quatro distritos ou microrregiðes: Ceres, Formosa, Jataí e Morrinhos. Nestas, as investigaçð̃es de rotina de captura e de determinação dos índices de infecção pelo Trypanosoma cruzi têm mostrado um crescimento progressivo da densidade e da infecção em Triatoma sordida, seguido pelo Rhodnius neglectus. Estas espécies se apresentam, no momento, como as mais importantes para a vigilância epidemiológica, uma vez que, a espécie T.infestans, reconhecidamente a mais importante na transmissão da doença de Chagas, praticamente está controlada no Estado de Goiás, com um resíduo no distrito de Formosa, no qual foram capturados 19 espécimes. Nos outros distritos, a captura não foi significativa, sendo um exemplar no distrito de Ceres e três em Jataí.

Os indices de infestação e de infecção de triatomíneos pelo T.cruzi, em Goiás, foram, respectivamente de: T.sordida 83.2 e 1,6\%; P.megistus 8,4 e $0,6 \% ;$ R.neglectus 7,3 e $0,2 \% ; T$. pseudomaculata 0,8 e $0,0 \% ; T$. infestans 0,3 e $0,0 \%$.

UNITERMOS: Triatomíneos, infecção, Trypanosoma cruzi, doença de Chagas.

\section{INTRODUÇÃO}

O risco de transmissão da doença de Chagas por triatomíneo está associado não só aos hábitos sinantrópicos e antropofilicos, como também aos índices de infestação e de infecção no ambiente domiciliar. Devido a estes fatores, em Goiás,

.. Depto. de Parasitologia - IPTSP/UFG - C.P.131 - Goiânia - Goiás.

- Técns.da Fund. Nac. de Saúde - convênio IPTSP/UFG - FNS

Recebido para publicação em 14/06/94 
ELIAS,C.N.;SILVA,I.G.da.;CAMARGO,M.F. \& ALVES,R.B.N. Índice de Infeç̧ão de triatomíneos pelo Trypanosoma cruzi, capturados no Ambiente Domiciliar no Estado de Goiás em 1993.Rev.Pat.Trop., $\quad 23$ ( 2 ):169-174, jul./dez.1994

Triatoma infestans era considerada a espécie, epidemiologicamente, mais importante, (LUSTOSA et al.1984a e b, SILVA, et al. 1992) e, até o momento, existem indícios de controle desta espécie, restando alguns bolsões de resíduos no estado, porém os exemplares capturados e examinados não apresentaram infecção. As espécies T.sordida e Rhodnius neglectus têm mostrado crescimento progressivo de infestação e de infeçyo pelo T.cruzi, no ambiente domiciliar,e isto aumenta o risco de transmissão, mesmo considerando as características das colônias, situadas predominantemente nos anexos, que, em Goiás, geralmente são contíguos ao domicílio.

$\mathrm{O}$ objetivo deste artigo é mostrar o crescimento progressivo da densidade e infecção das espécies T.sordida e R.neglectus, que apresentavam até pouco tempo, características marcadamente peridomiciliares, e que no momento, têm aumentado a frequêencia no intradomicílio, sinalizando uma possibilidade de substituir a espécie controlada, T.infestans.

\section{MATERIAL E MÉTODOS}

A partir da captura de triatomíneos no ambiente domiciliar (domicílio e anexos), no Estado de Goiás, os insetos eram remetidos ao laboratório para serem identificados ao nível de espécie e examinados para se determinar o índice de infecção natural pelo T.cruzi. Os exames eram feitos colhendo-se por compressão do abdome, as fezes, homogeneizadas em solução fisiológica $(0,9 \%)$, e examinadas ao microscópio com médio aumento $(400 \mathrm{X})$. De cada triatomíneo infectado (positivo) faziam-se 3 lâminas de acordo com a sezuinte metodologia: misturava-se ao material fecal com T.cruzi, uma gota de solução fisiológica $(0,9 \%)$ contendo soro humano (preparada pela mistura de $25 \mathrm{ml}$ da solução com $5 \mathrm{ml}$ do soro); duas gotas desta mistura eram retiradas e transferidas para lâminas linpas, fixadas com álcool metilico (96\%); após a secagem a lâmina era imersa no azul de metileno e, em seguida, lavada em água destilada e corada pelo Giemsa (30'), e examinada ao microscópio com a imersão (100x).

\section{RESULTADOS}

Os resultados são apresentados nas Tabelas 1, 2, 3, 4, e 5. Os índices de infestação e de infecção de triatomíneos pelo T.cruzi, em todo Estado de Goiás, no ano de 1993, encontram-se em ordem decrescente: T.sordida - 83,2 e 1,6\%, P.megistus 8,4 e $0,2 \%$, R.neglectus - 7,3 $€ 0,2 \%$, P.pseudomaculata - 0,8 e $0,0 \%$, e T.infestans $-0,3$ e $0,0 \%$.
ELIAS,C.N.;SILVA,I.G.da.;CAMARGO,M.F. \& ALVES,R.B.N. Índice de Infeç̧ão de triatomineos pelo Trypanosoma cruzi, capturados no Ambiente Domiciliar no Estado de Goiás em 1993.Rev.Pat.Trop., $\quad 23$ ( 2 ):169-174, jul./dez.1994

A espécie T.infestans está controlada em Goiás (como mostram as Tabelas 1 , $2,3,4$ e 5) nas quais as capturas mostraram a presença de triatomíneo apenas de forma residuária. Devido às suas características estritamente domiciliares e por não ter foco silvestre desse triatomíneo no Brasil, esta espécie pode ser erradicada, dependendo apenas das ações de controle e de vigilância.

Tabela 1 - Índices de infestação e de infecção de triatomíneos, pelo Trypanosoma cruzi, no ambiente domiciliar em Goiás, no ano de 1993.

ESPÉCIE

IE
INFESTAÇÃO(\%) INFECÇÃO(\%)

\begin{tabular}{lcc}
\hline Triatoma sordida & 83,2 & 1,6 \\
Panstrongylus megistus & 8,4 & 0,6 \\
Rhodnius neglectus & 7,3 & 0,2 \\
Triatoma pseudomaculata & 0,8 & 0,0 \\
Triatoma infestans & 0,3 & 0,0 \\
\hline
\end{tabular}

Tabela 2 - Frequência de triatomíneos no ambiente domiciliar e infeç̧ão pelo Trypanosoma cruzi, na microrregião de Ceres - Goiás, no ano de 1993.

TRIATOMÍNEOS

ESPÉCIES

CAPTURADOS EXAMINADOS POSITIVOS

\begin{tabular}{lcccc} 
& & & INFECÇÃO \\
\hline Triatoma sordida & 466 & 409 & 01 & 0,24 \\
Panstrongylus megistus & 350 & 298 & 03 & 1,01 \\
Rhodnius neglectus & 149 & 138 & 0,0 & 0,00 \\
Triatoma pseudomaculata & 55 & 53 & 0,0 & 0,00 \\
Triatoma infestans & 01 & 01 & 0,0 & 0,00 \\
\hline
\end{tabular}

Tabela 3 - Frequência de triatomíneos no ambiente domiciliar e infecção pelo Trypanosoma cruzi, na microrregião de Formosa - Goiás, no ano de 1993.

TRIATOMÍNEOS

ESPÉCIES

\begin{tabular}{ccc}
\hline CAPTURADOS EXAMINADOS POSITIVOS & $\begin{array}{c}\text { \% DE } \\
\text { INFECCAXO }\end{array}$
\end{tabular}

Triatoma sordida

1926
03
137
19

1741

14

0,80

Panstrongylus megistus

Rhodnius neglectus

1741
01
132
18

Triatoma costalimai

09

18

Tabela 4 - Frequência de triatomíneos no ambiente domicil microrregião de Morrinhos - Goiás, no ano de 1993. 
ELIAS,C.N.;SILVA,I.G.da.;CAMARGO,M.F. \& ALVES,R.B.N. Índice de Infeç̧ão de triatomíneos pelo Trypanosoma cruzi, capturados no Ambiente Domiciliar no Estado de Goiás em 1993.Rev.Pat.Trop., $\quad 23$ (2):169-174, jul./dez.1994

\section{TRIATOMÍNEOS}

\begin{tabular}{lcccc} 
ESPÉCIES & CAPTURADOS & EXAMINADOS & POSITIVOS & $\begin{array}{c}\text { \% DE } \\
\text { INFECÇÃO }\end{array}$ \\
\cline { 2 - 5 } & 3.530 & 2.451 & 70 & 2,85 \\
\hline Triatomo cordida & 242 & 240 & 07 & 2,91 \\
Pansırongylus megistus & 90 & 71 & 00 & 0,00 \\
Rhodnius neglectus & 14 & 14 & 00 & 0,00 \\
Triatoma pseudomaculata & & &
\end{tabular}

Tabela 5 - Frequência de triatomíneos no ambiente domiciliar e infecção pelo Trypanosoma cruzi, na microrregião de Jataí - Goiás, no ano de 1993

\section{TRIATOMINEOS}

\begin{tabular}{lcccc} 
ESPÉCIES & CAPTURADOS & EXAMINADOS & POSITIVOS & $\begin{array}{c}\text { \% DE } \\
\text { INFECÇÃO }\end{array}$ \\
\hline Triatoma sordida & 1.557 & 788 & 0,0 & 0,0 \\
Panstrongylus megistus & 13 & 04 & 0,0 & 0,0 \\
Rhodnius neglectus & 255 & 130 & 0,0 & 0,0 \\
Triatoma pseudomaculata & 01 & 01 & 0,0 & 0,0 \\
Triatoma infestans & 03 & 03 & 0,0 & 0,0 \\
\hline
\end{tabular}

\section{DISCUSSÃO}

Das espécies sinantripicas de triatomíneos, T.infestans era considerada, epidemiologicamente, a mais inportante no Estado de Goiás, apresentando uma vasta distribuição geográfica tendo sido controlado em 112 municípios dos 173 investigados. $\mathrm{O}$ índice de infecção natural de T. infestans pelo T.cruzi era de $2,21 \%$ na década de 70 (LUSTOSA et al., 1984b) € na década seguinte, até 1988, apresentou uma distribuição maior, estando presente em $68,21 \%$ dos municípios, com uma frequência domiciliar de $10,70 \%$ e $2,64 \%$ de infecção natural pelo T.cruzi (SILVA et al.,1992).

Os trabalhos de combate ao vetor, que se mostraram eficientes para o intradomicílio, praticamente coitrolaram o $T$. infestans e isto pôde ser verificado neste levantamento, no qual esta espéie apresentou índice de infestação domiciliar de 0,3\%, sem infeccão pelo T.cruzi, comdistribuição residual na microrregião de Formosa. Nas
ELIAS,C.N.;SILVA,I.G.da.;CAMARGO,M.F. \& ALVES,R.B.N. Indice de Infeç̧âo de triatomineos pelo Trypanosoma cruzi, capturados no Ambiente Domiciliar no Estado de Goiás em 1993.Rev.Pat.Trop., 23 (2):169-174, jul./dez.1994

outras microrregiões do Estado de Goiás o T.infestans está controlado. Na microrregião de Ceres, com relação ao município de Pirenópolis, os dados são concordantes com os de SILVA et al.(1993).

Com relação ao peridomicílio, as ações de combate ou controle não se mostraram eficientes, e este é o maior desafio atualmente para adequar ou estudar medidas para atingir os triatomíneos nos anexos do domicílio, associados à modificação dos costumes e participação dos habitantes. Os problemas do peridomicílio são a diversidade e proximidade deles ao domicílio, tendo servido não só de mecanismo de escape do triatomíneo ao inseticida, como também um lugar que protege o inseto e permite recrudescer as colônias de triatomíneos e reinfestar tanto o peridomicílio quanto o domicílio, pela dispersão ativa quando a colônia atinge um número elevado de triatomíneos. Na maioria dos casos, os triatomíneos não têm nenhum trabalho em atingir o intradomićlio devido à contigüidade entre estes ambientes.

As espécies T.sordida e R.neglectus merecem atenção por terem mostrado um crescimento progressivo da densidade no ambiente domiciliar, trazendo a perspectiva de ocuparem os espaços nas regiões onde o T.infestans foi controlado. Um fato que chamou a atenção foi o índice elevado da infecção natural de T.sordida pelo T.cruzi, de $1,6 \%$, determinado neste trabalho em relação a outros, nos quais os índices encontrados foram de $0,47 \%$, no período de $1976 / 80$ (LUSTOSA et al., 1984) e de $1,13 \%$, no período de 1984/88 (SILVA et al., 1992).

A precariedade das habitações rurais (ranchos, cafuas, casebres e outros) e a proximidade do intradomicílio com os anexos, têm facilitado a invasão de T.sordida à habitação humana. Em Goiás, o paiol é o anexo mais importante na manutenção e colonização de triatomíneos, por apresentar-se quase sempre contíguo ao galinheiro e ao intradomicílio. Esta situaçao, favorece a permanência do triatomíneo nesse anexo, porque as aves garantem a alimentação, ao passo que as espigas não se acomodando perfeitamente, deixam espaços onde os triatomíneos se abrigam ficando protegidos da ação dos inseticidas e de outros predadores. O paiol do produtor de milho tem servido também de centro de dispersão passiva de T.sordida, este triatomíneos chega ao paiol do consumidor através do milho adquirido, como mostra o trabalho de SILVA et al.(1993). O R.neglectus tem apresentado pequenas colônias nos anexos, evidenciando menor grau de adaptação do que o T.sordida. O intradomicílio é geralmente invadido por esse triatomíneo, pela dispersão ativa, ocorrendo devido à proximidade da habitação e ao meio silvestre. Nas pequenas cidades, a invasão dessa espécie ocorre inicialmente pelas ruas periféricas, onde tem sido capturada com maior frequência. Será necessário, no entanto, estudo de novas estratégias de controle de triatomíneos no peridomicilio, assim como, de outras formulações de inseticidas que possibilitem alcançá-los nesses anexos e controlá-los. 
ELIAS,C.N.;SILVA,IG.da.;CAMARGO,M.F. \& ALVES,R.B.N. Índice de Infecção de triatomíneos pelo Trypanosoma cruzi, capturados no Ambiente Domiciliar no Estado de Goiás em 1993.Rev.Pat.Trop., 23 ( 2 ):169-174, jul./dez.1994

\section{SUMMARY}

Triatomines' infection ratios by Trypanosoma cruzi captured in the domiciliary environment in State of Goiás in 1993.

In State of Goiás, researches carried of routine domestic searches about vectors Chagas' disease, were carried in four district: Ceres, Formosa, Jataí e Morrinhos. The searches have showed growth progressive of density and infection of Triatoma sordida and Rhodnius neglectus by Trypanosoma cruzi. These species are the most importants in surveillance epidemiologic, because T.infestans, the vectors most important Chagas' disease transmission is controled.

The triatomines' ratios infestation domiciliary and infection by T.cruzi, were, respectively of: T.sordida - 83.3 and $1.6 \% ; P$. megistus - 8.4 and $0,6 \%$; R.neglectus 7.3 and $0.2 \%$; T. pseudomaculata -0.8 and $0.0 \%$; T.infestans -0.3 and $0,0 \%$.

KEYWORDS: Triatomines, infection, Trypanosoma cruzi, Chagas'disease.

\section{REFERÊNCIAS BIBLIOGRÁFICAS}

01. LUSTOSA, E. de S.; NAVES, H.A.M.; CARVALHO, M.E.S.D.; CALIL, F. \& MATOS, C.A. de S. Distribuição geográfica encontrados nos domicílios e peridomicílios nas micro-regiões do estado de Goiás. Rev.Goiana Med.,30:49-54, 1984a.

02. LUSTOSA, E. de S.; NAVES, H.A.M.; CARVALHO, M.E.S.D.; CALIL, F. \& MATOS, C.A. de S. determinação dos índices de infecção natural por Trypanosoma cruzi em Triatomíneos nas 16 microrregiões do estado de Goiás. Rev. Goiana Med.,30: 153-155, 1884.

03. SILVA, I. G. da; ISAC, E.; NAKANO, H.; SILVA, E.; MOURA, A.F.;SILVA,J.L. \& QUEIRÓZ, A.L. feito residual da deltametrina (K- Othrine Flow 50) no controle de triatomíneos em Pirenópolis,Goiás. Rev.Pat.Trop.,22:253257,1993 .

04. SILVA. I.G. da; SILVA, J.L.; SILVA. H.H.G. da; CAMARGO, M.F.; MOURA, A.F.; ELIAS.M.; \& SANTOS, A.H. Distribuição dos vetores da Triapanosomíase americana capturados no ambiente domiciliar no estado de Goiás, no período de 1984/88. An.Soc.ent.Brasil, 21:139-154, 1992. 